\title{
INHIBITION IMMÉDIATE DE L'INFECTIVITÉ DES GAMETOCYTES DE PLASMODIUM YOELII NIGERIENSIS PAR LE SÉRUM DE RONGEURS INFECTÉS DEPUIS 5 JOURS
}

\author{
G. PETIT*, D. CAMUS**, E. DEI-CAS** et I. LANDAU*
}

Au cours de nombreuses infections malariennes, l'infectivité des gamétocytes diminue ou s'annule alors que leur production est particulièrement forte (Vanderberg et Gwadz, 1980). Ainsi au cours de l'infection expérimentale standard de souris blanches par Plasmodium yoelii nigeriensis, le sang atteint le maximum d'infectivité pour les anophèles le $3^{\mathrm{e}}$ jour, au moment où les gamétocytes sont relativement peu abondants dans le sang périphérique; paradoxalement cette infectivité s'annule dès le $5^{\mathrm{e}}$ jour, moment où les gamétocytes deviennent très abondants dans le sang périphérique (Landau et coll., 1979).

L'expérience suivante réalisée avec Plasmodium yoelii nigeriensis, chez la souris blanche, montre que cette perte d'infectivité est liée à l'apparition d'un facteur sérique :

- 6 souris reçoivent chacune en IV $0,07 \mathrm{ml}$ de sang provenant d'une souris infectée depuis 3 jours. Trois de ces souris $(A, B$ et $C)$ reçoivent immédiatement après en IV $0,5 \mathrm{ml}$ de sérum provenant de souris saines (lot témoin); les 3 autres (D, E et F) reçoivent $0,5 \mathrm{ml}$ de sérum provenant du sang de souris au $5^{\mathrm{e}}$ jour d'infection.

- Une demi-heure après cette double injection, 20 anophèles sont gorgés sur chaque souris ; l'infectivité développée (estimée par le comptage des oocystes 7 jours après le repas) est représentée dans le tableau $I$.

TABLEAU I. - Nombre moyen d'oocystes par anophèles.

\begin{tabular}{lccc}
\hline Souris & A & B & C \\
Sérum sain & $15,6 \pm 18,2^{*}$ & $19,3 \pm 19,6$ & $23 \pm 24,3$ \\
\hline Souris & D & E & F \\
Sérum 5 J & 0 & $1,16 \pm 1,4$ & $0,17 \pm 0,3$ \\
\hline
\end{tabular}

* Erreur standard.

* Laboratoire de Zoologie (Vers), associé au CNRS, Muséum National d'Histoire Naturelle, 61, rue, Buffon, F 75231 Paris Cedex 05.

** Unité 42 (INSERM), Domaine du Certia, 369, rue Jules Guesde, F 59650 Villeneuve-d'Ascq. 
Le sérum des souris infectées depuis 5 jours inhibe donc in vivo, l'infectivité des gamétocytes de Plasmodium yoelii nigeriensis.

Le modèle expérimental utilisé ici, et que nous détaillerons par ailleurs, nous paraît bien adapté à l'étude approfondie des mécanismes de blocage de l'infectivité des gamétocytes chez les Plasmodiums.

\section{BIBLIOGRAPHIE}

Landau I., Miltgen F., Boulard Y., Chabaud A. G., Baccam D. - Études sur les gamétocytes des Plasmodiums du groupe "vivax ": morphologie, évolution, prise par les anophèles et infectivité des microgamétocytes de Plasmodium yoelii. Ann. Parasitol. Hum. Comp., 1979, s4, I45-I6I.

VANDERBERG J. P. et GWADz W. R. - The transmission by mosquitoes of Plasmodia in the laboratory, in: Malaria, vol 2, Pathology vector Studies and culture, 328 p., ed. Julius. P. Kreier, Academic Press, Londres, 1980. 